\title{
How are vehicles running in underground expressways?
}

\author{
Y. Wang, Y. Liu \& S. Zheng \\ Key Laboratory of Road and Traffic Engineering \\ of the Ministry of Education, Tongji University, China
}

\begin{abstract}
With advantages of less land use and better protection for urban landscapes, the underground expressway has become a new solution for urban traffic problems. Relevant safety studies are deficient despite of their significance in urban road networks. The differences between expressways on and under the ground made it inappropriate to apply the detailed design of the former directly or mechanically to the latter. In order to improve transport safety in underground expressways, the operational characteristics of vehicles in expressways were thoroughly compared. Natural driving experiments were carried out in three underground expressways in Shanghai, China. Traffic flow data and lane information were obtained from the Monitoring Center and the Tunnel Management Center by using detection coils and gun-shaped HD cameras respectively. Speed, headway and lateral movement were chosen as key indicators of vehicle characteristics. The results of research on their relations not only reveal how expressways are different, but also emphasize transport safety problems and potential risks in car-following scenarios. It is also concluded from vehicle track analysis that instead of symmetrical distribution, the distance to the right-side wall for vehicles running on the right lane is $0.55 \mathrm{~m}$ greater (at $95 \%$ confidence level) than the distance to the left-side wall for vehicles running on the left lane. Therefore, taking into account its own width, a vehicle running in the right lane has the tendency to invade the left lane, increasing the risk of lateral collision. Suggestions were also made on safety improvement.
\end{abstract}

Keywords: underground expressway, visual environment, vehicle running characteristics, car-following scenario. 


\section{Introduction}

As modern cities continue to expand with increasing traffic density, diverting urban transport to underground space has become a new trend for intensified urban development since large-scale transport network construction not only leaves urban traffic problems unresolved but brings about serious environmental issues. Space resources got further consumed in rapid urban development and underground space exploitation became a necessary trend. Safety issues start to draw increasingly more attention since complement of underground road system construction has been on agenda.

Although the construction of the first subway in London 150 years ago marked the start of human's effort to make use of underground space for transport, the development of underground roads is slow and few system networks has been constructed due to the fact that urban traffic in developed countries is approaching stable equilibrium thus no strong demand for underground road network is expected. In spite of its merits of less land use and better protection for urban landscape, underground expressway had not been put into operation in developed countries until 1990s and it is still a new road type worthy of exploration. Currently underground expressway has become an important part of transportation system in metropolitans like Boston and Singapore $[1,2]$.

Nowadays, China's population is shifting rapidly to expanding urban areas. With active economic and cultural activities, urban residents constantly increase their private car ownership and travel frequency, creating higher transport demands. Despite a late start, underground transport projects have been developed in a stunning speed in China, such as the Well-Shaped Channel in Shanghai and the Four Vertical and Two Horizontal Underground Expressway Network in Beijing [3]. However, the planning and design of these underground expressways are still based on existing design standards and references for general urban expressways and it is not guaranteed that normative standards could be reasonably applied to underground facilities.

Aiming at providing better service for urban transport, this article firstly presents comparisons of vehicle characteristics and visual environment between expressways on and under the ground, and then focuses on how vehicles are running in expressways, taking into considerations of drivers' speed choice and other driving behaviors in both car-following scenario and free-flow scenario.

\section{Underground expressway}

\subsection{Definition}

An expressway is an access-controlled road type in an urban area, with a central median, two-lane or multi-lane in one way and equipped with transport safety facilities and management facilities. Combining features of urban main road and freeway, expressway has design speed and capacity between them. Its essential 
function is to establish rapid and direct transport links between urban districts by separating long-distance traffic flow and regional traffic flow. It is also effective in mitigating urban traffic congestion, improving living environment and promoting sustainable urban development.

In this article, underground expressway is defined as fully sealed expressway constructed in underground space. Its advantages include better protection of original urban landscape, more effective utilization of land resources and less affected by external environment, for example, adverse weathers conditions like raining, snowing, and fog. Besides, in comparison with subway, much less investment would be involved in construction of underground expressway [4].

\subsection{Environmental features}

Expressways on and under the ground have similar access control, traffic flow and separation. Meanwhile, from driver's view, differences between them mainly reveal in three aspects: illumination, travel space and roadside reference.

In general, human's sight adapt well to natural light when driving during the daytime. While artificial illumination must be applied to underground expressway due to the lack of natural light. The light source of artificial illumination is mainly high-pressure sodium or fluorescent lamps, which creates visual environment either pre-yellow or pre-white in comparison with natural light. Inadequate illumination increases the difficulty and insensitivity for the driver to identify objects in front of the running vehicle, including obstacles and other vehicles. And the sudden change between natural light and artificial illumination at entrances and exits leads to driver's visual discomfort and blur: a sudden dark visual environment at entrances of underground expressways may lead to Black Hole effect while a sudden bright visual environment at exits may result in glare effect. In an area where tunnels are as safe as high standard modern roads, crash rate would be higher at entrance zone and crash severity would be higher in tunnels [5].

Underground expressway could be seen as a pipeline accommodating a number of vehicles, with height constraints and lane restriction. The limited space requires that traffic signs with sufficient information and legibility in limited layout be installed within controlled height [6]. Meanwhile, it is worth noting that long-distance travel in an enclosed space has a negative psychological impact on the driver.

In an open space, landscape offers additional information for the driver besides its positive effect on visual adjustment to environment. It even helps in predicting upcoming road geometry. Meanwhile, the monotonous concrete tunnel wall of underground expressway provides little support in such aspects. Because of insufficient roadside reference, drivers in underground expressways are more likely to make mistakes in exit choice and speed choice thus resulting in higher driving risk. 


\section{Method}

\subsection{Characteristics indicators}

Vehicle operating characteristics include speed, acceleration, deceleration, headway, etc. [7]. Monotonous closed driving environment and limited view in underground expressways lead to unique vehicle operating characteristics. To conduct comparative analysis, relevant experiments are required to be specific and adaptable. In this article, speed, headway and lateral space were selected as key indicators for vehicle characteristics. (1) Speed is the average speed representing general vehicle operating features. It is the main crash cause, direct behavior display of driver's perception of driving environment and the basis for further studies. (2) Headway refers to the gap between rear of the front vehicle and head of the following vehicle. It reflects driver's perception of running speed, braking distance and how the driver handles dynamic distance to the front vehicle. (3) Lateral space refers to the distance from the edge of vehicle near roadside on that side to roadside objects, such as fence for elevated expressway, noise proofing walls for expressway on the ground and sidewall for underground expressway.

\subsection{Data collection}

Traffic flow data and lane information were obtained from relevant administrative departments-Monitoring Center and Tunnel Management Center, through coil detection and surveillance video analysis. 10,275 groups of data for test sections of underground expressways and 18,602 data groups of the West section of the Mid-Ring Expressway were collected in 2 consecutive sunny days.

In natural driving experiments, space and speed information was collected by laser rangefinder and non-contact speedometer, which was locally called the five-wheel instrument and then integrated into vehicle operating characteristics. Test sites include Xinjian Road Tunnel, Renmin Road Tunnel and West section of Mid-Ring Expressway in Shanghai. Xinjian Road Tunnel and Renmin Road Tunnel were newly built tunnels with multiple entrances and exits. As main passages to cross Huangpu River, they were categorized as underground expressways, with high traffic flow in peak hour. The West section of the Mid-Ring Expressway of Shanghai was chosen for comparison on account of its similar profile. It was typical urban expressway consisting of elevated part and other parts on the ground.

Three groups of experiments were carried out in underground expressways to evaluate vehicle operation: (1) enter in ramp and exit in main line, (2) enter in main line and exit in ramp, and (3) enter in main line and exit in main line. The routes of natural driving experiments are illustrated in Figure 1.

21 subjects participated in this natural driving experiment, each of whom drove through 3 complete routes on roadmaps in the same order. Dynamic information on vehicle characteristics in test sections of expressways was obtained. Questionnaire was also carried out to better understand drivers' subjective feelings. 
(1)

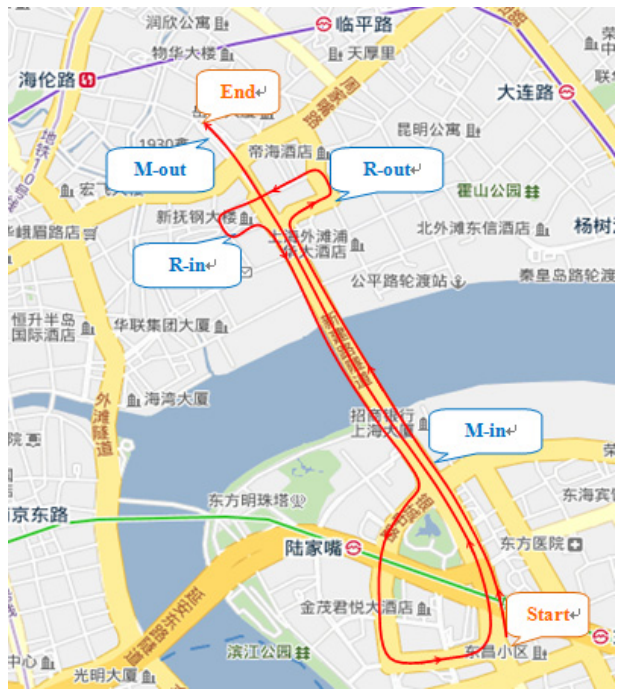

(2)

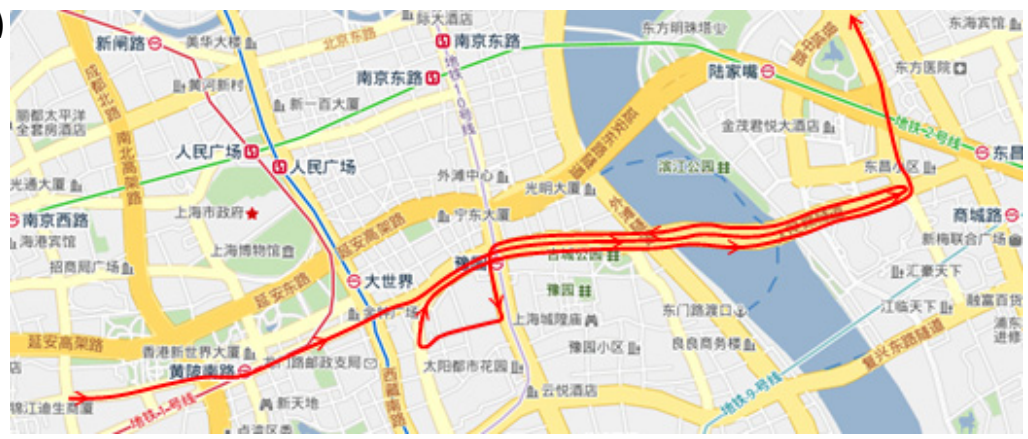

(3)

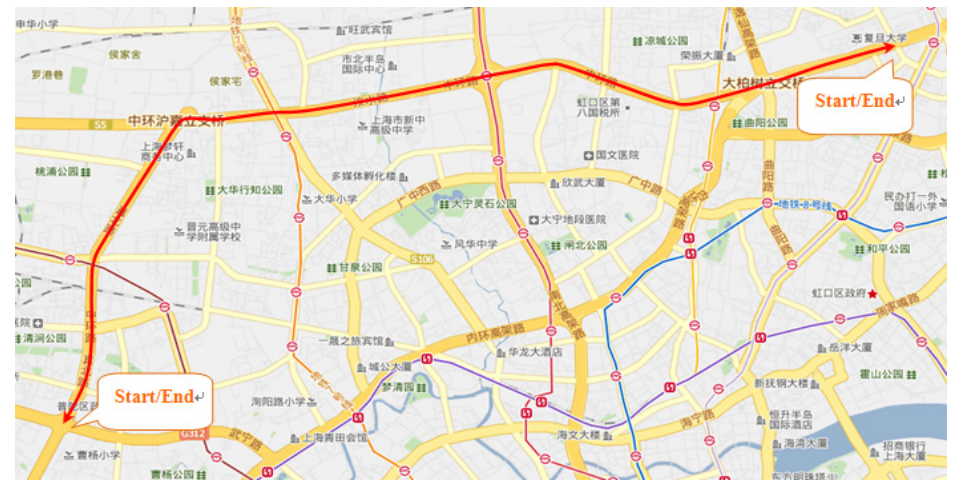

Figure 1: Natural driving routes in roadmaps: (1) Xinjian Road Tunnel, (2) Renmin Road Tunnel, (3) West section of Mid-Ring Expressway. 


\section{Analysis}

It is acknowledged that the critical headway is $150 \mathrm{~m}$. The traffic flow with headway greater than that is seen as free flow while the traffic flow with headway less than that would be car-following scenario [8].

In free flow scenario, traffic flow density is relatively small and driving is performed mainly based on vehicle condition, road conditions and driver's characteristics such as driving skills, personality, physical and physiological condition. In such case, desired speed could be maintained as driving is little unaffected by other road users.

In the car-following scenario, higher traffic flow density leads to headway reduction, while speed of the following vehicle is severely constrained by speed and other behaviors of the front vehicle. In such case, the driver of the following vehicle has to balance between saving travel time and avoiding collision and simultaneously keep driving in a corresponding speed.

Statistical independence test was carried out to define whether certain correlation exits among three selected indicators.

\subsection{Speed and headway}

\subsubsection{Speed - headway independence test}

For expressways, main line was chosen as the target segment for this study because the circumstances that headway goes beyond the critical value $150 \mathrm{~m}$ mostly occurs in the main line. Taking speed as variable A, headway as variable $\mathrm{B}$, for headways either above or below $150 \mathrm{~m}$, Speed-Headway independence test was carried out. Abnormal data in cases that speed is greater than $120 \mathrm{~km} / \mathrm{h}$ or headway is greater than $5000 \mathrm{~m}$ was excluded.

In the test, speed is divided into six groups by step length of $20 \mathrm{~km} / \mathrm{h}$; while headway is divided into five groups by step length of $30 \mathrm{~m}$ when it is not above $150 \mathrm{~m}$ and seven groups by step length of $50 \mathrm{~m}$ when goes beyond that. The results are shown in Table 1.

Table 1: Speed - headway independence test results.

\begin{tabular}{|c|c|c|c|}
\hline Sample & & $\mathrm{X}^{2}$ & $(\mathrm{~m}-1)(\mathrm{k}-1)$ \\
\hline \multirow{2}{*}{ Expressway } & Headway $<150 \mathrm{~m}$ & 2524.2 & 20 \\
\cline { 2 - 4 } & Headway $>150 \mathrm{~m}$ & 46.33 & 30 \\
\hline \multirow{2}{*}{$\begin{array}{c}\text { Underground } \\
\text { expressway }\end{array}$} & Headway $<150 \mathrm{~m}$ & 108.47 & 20 \\
\cline { 2 - 4 } & Headway $>150 \mathrm{~m}$ & 28.06 & 30 \\
\hline
\end{tabular}

It can be learned from Table 1 that at $\mathrm{a}=0.01$, for expressways of either type, variables are related in car-following scenario since $\mathrm{X} 2>\mathrm{X} 20.01(20)=37.566$, they are not significantly related in free flows because $\mathrm{X} 2<\mathrm{X} 20.01(30)=50.892$. 


\subsubsection{Car-following scenario}

Vehicle running speed generally changes in a regularity that less traffic volume and density leads to higher speed and vice versa. However, in similar conditions of traffic volume and density, speed distributions could be remarkably different for variety of road types, for which the deciding factor is driving environment [9].

In a car-driving scenario, speed observation needs to be integrated with headway or it would be left meaningless. In order to reflect characteristics of natural light for expressways on the ground, data for analysis was collected during the daytime (7:00-17:00). Data in grade separation sections was excluded on account of unstable speed in such sections.

For the situation in which headway is less than $150 \mathrm{~m}$, a scatter plot is obtained as below (Figure 3) with speed shown in the vertical bar and headway in the horizontal bar.

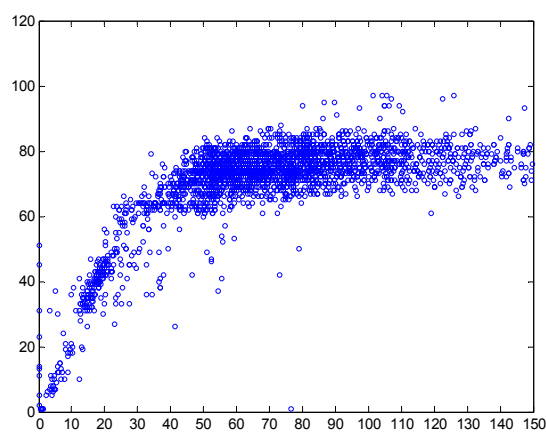

(1)

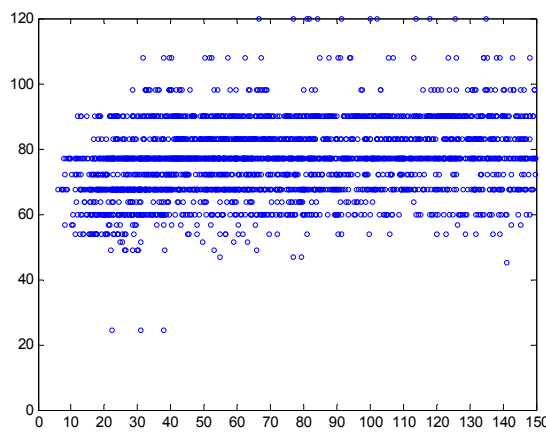

(2)

Figure 2: Speed - headway scatter plot for straight sections: (1) Expressway main line, (2) Underground expressway main line.

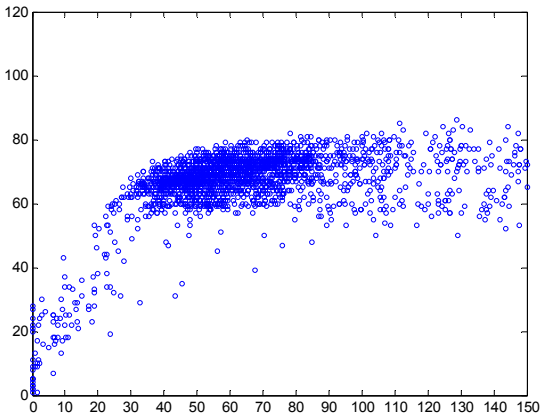

(1)

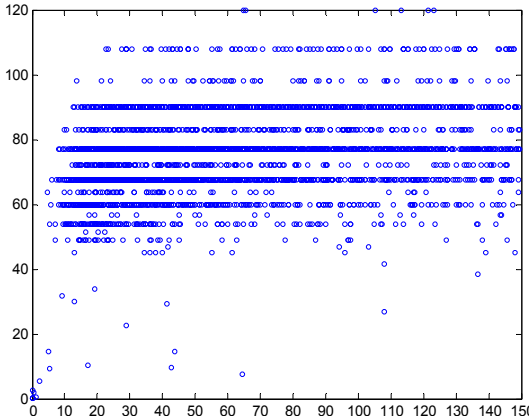

(2)

Figure 3: Speed - headway scatter plot for curved sections: (1) Expressway main line, (2) Underground expressway main line. 
Figure 3 displays that on the main line of expressway speed generally increases with space in a linear trend until space reaches a certain value. Then the speed increase slows down and settles at a certain value consistent with corresponding speed limit $80 \mathrm{~km} / \mathrm{h}$. Meanwhile, it also could be learned that the relation between speed and headway was not so obvious for underground expressways. Vehicle's running speed basically stays between $60 \mathrm{~km} / \mathrm{h}$ and $90 \mathrm{~km} / \mathrm{h}$, which is far beyond the site speed limit $40 \mathrm{~km} / \mathrm{h}$ and even greater than the speed limit for expressways at some locations.

The results could be explained by that human's perception of speed declines in environment under the ground. In this situation the driver has the tendency to fail to perceive rising driving risk brought about by unaware speeding, especially in a short headway. Besides, with such an illusion of safety, the driver is likely to keep driving in a speed not adept well to the driving environment.

\subsection{Lateral Space}

\subsubsection{Speed - Lateral Space independence test}

In the Speed-Lateral Space independence test, speed is divided by step length of $5 \mathrm{~km} / \mathrm{h}$; while the stable distance to side wall is divided by step length of $0.5 \mathrm{~m}$. Results are shown in Table 2.

Table 2: Speed - Lateral Space independence test results.

\begin{tabular}{|c|c|c|c|}
\hline Sample & & $\mathrm{X}^{2}$ & $(\mathrm{~m}-1)(\mathrm{k}-1)$ \\
\hline \multirow{2}{*}{ Expressway } & Left & 5.3023 & 9 \\
\cline { 2 - 4 } & Right & 1.6961 & 6 \\
\hline \multirow{2}{*}{$\begin{array}{c}\text { Underground } \\
\text { expressway }\end{array}$} & Left & 2.9966 & 6 \\
\cline { 2 - 4 } & Right & 21.152 & 15 \\
\hline
\end{tabular}

At a $=0.01, \mathrm{X} 20.01(6)=16.812, \mathrm{X} 20.01(9)=21.666, \mathrm{X} 20.01(15)=30.578$. It could be obtained after comparison that variables are not significantly related and therefore could be seen as independent from each other.

\subsubsection{Free running state}

Environment's impact on lateral space includes that interference at one side may lead the driver to turn the wheel to the other side. The distance from vehicle's edge to the side wall (on the same side) when 21 subjects were driving was recorded. Data in weaving sections and curved sections was exempted to eliminate the effects brought about by lane change behavior. In total, 44,607 track points were collected. When the data analysis suggested that lateral space in underground expressway is asymmetric, further steps were taken to explore the reason and mechanism.

TJRD vehicle tracking software was applied to track trajectories of vehicles. In order to conduct quantitative analysis, each lane was divided into ten sub-lanes by step width of $0.35 \mathrm{~m}$. 


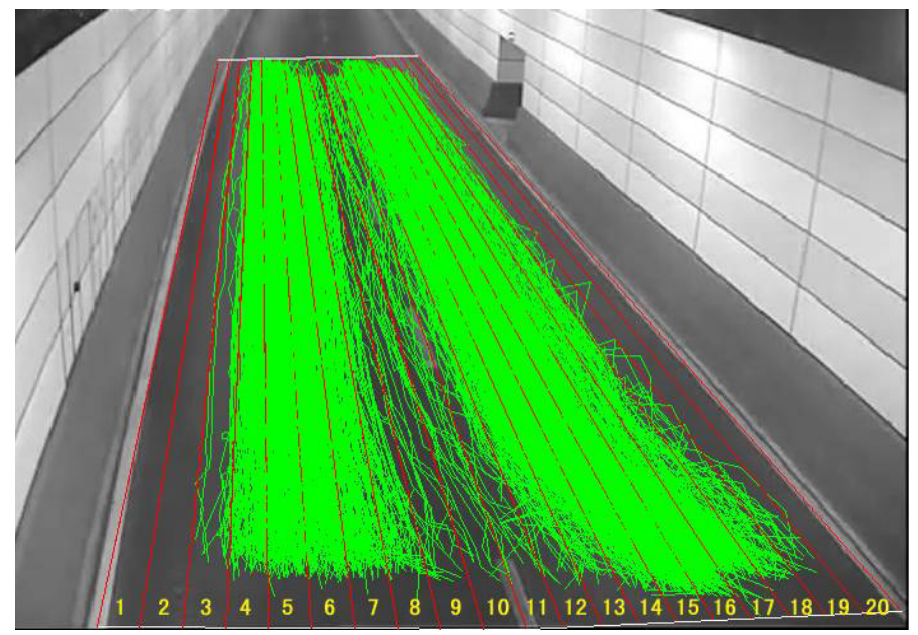

Figure 4: Sub-lane partition in an underground expressway.

The number of vehicle trajectory on each sub-lane was recorded and statistical frequency histogram was generated. Normal distribution fitting was also displayed in Figure 5 below.

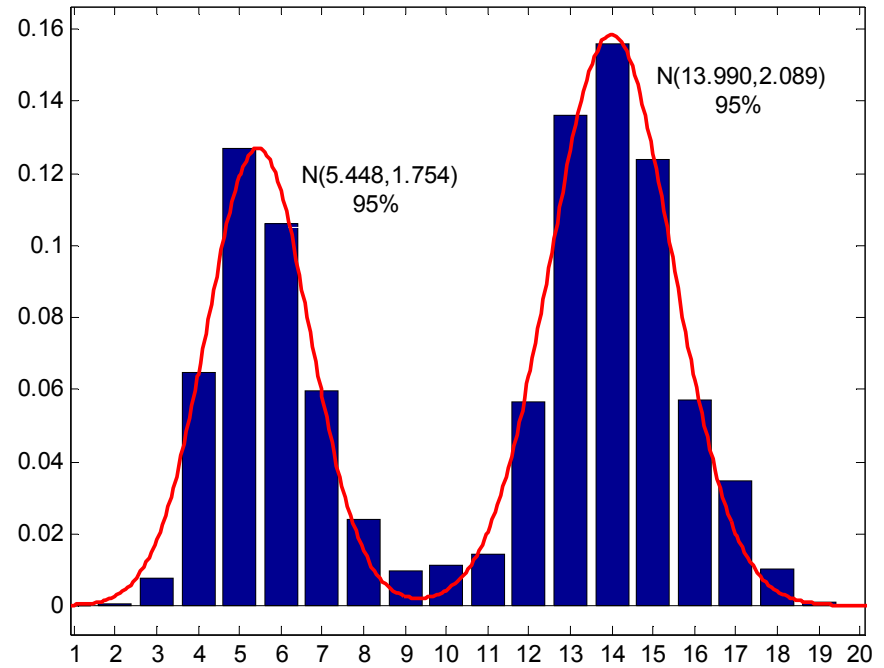

Figure 5: Statistical frequency histogram and normal distribution fitting.

Due to specific sub-lane partition, for a track point in zone $n$, its distance to the left wall could be approximately calculated by the function below:

$$
\mathrm{X}_{\mathrm{L}}(\mathrm{i})=(\mathrm{n}-1) \times 0.35+\mathrm{a}(\mathrm{i}) \times 0.35
$$


where, XL(i) is the distance of $\mathrm{i}$ th track point to the left wall;

$\mathrm{N}$ is the zone $\mathrm{i}$ th track point belongs to;

$a(i)$ is the distance of $i$ th track point to the left dividing line of its zone.

Similarly, its distance to the right wall:

$$
\mathrm{X}_{\mathrm{R}}(\mathrm{i})=(20-\mathrm{n}) \times 0.35+\mathrm{b}(\mathrm{i}) \times 0.35
$$

where, $\mathrm{XR}(\mathrm{i})$ is the distance of $\mathrm{i}$ th track point to the right wall;

$b(i)$ is the distance of $i$ th track point to the right dividing line of its zone.

It is displayed in the figure that track points of vehicles are mainly concentrated in the middle of each lane, with crest of distance from right edge to right side wall greater than crest of distance from left edge to left side wall by approximately two step widths. In normal distribution fitting at confidence level of $95 \%$, mean position track of left tires is at step 5.448, representing that the mean distance from left edge to left side wall is $1.907 \mathrm{~m}$, while mean position track of right tires is at step 13.990, representing that the mean distance from left edge to left side wall is $2.454 \mathrm{~m}$. There is a difference of $0.547 \mathrm{~m}$ between these two values. Taking into account vehicle's own width, it is reasonable to deduce that vehicles running on the right lane of underground expressways have the tendency to cross the lane marking even invade into the left lane, which increases the risk of lateral collision. This could be explained by that since drivers sit at left in China, they acknowledge road and environment condition on the left side better than the right side, which gives them more confidence to estimate the distance at left when they are controlling the running vehicle. This also explains why the driver is more inclined to keep the track away from the right side wall when driving on the right lane.

It is worth noting that when vehicles are running in expressways on the ground, there is no significant difference between the distances from vehicle's edge to road fence on the adjacent side. Lateral space information indicates that the impact of guardrail or fence is much smaller than side wall in driving environment.

\section{Conclusion}

This paper explores environmental differences between expressways on the ground and expressways built in underground space. With traffic flow data and vehicle operating characteristics data collected from natural driving experiments, including speed, headway and lateral space, this study concludes from statistical analysis that driving behaviour resulted from the special visual environment in underground expressways increases driving risk through highly discrete running speed, frequent speeding and vehicle running on the right lane getting across lane marking.

The core idea of suggestions for improvement of road safety in underground expressways is to make driving environment better and road geometry more consistent. Better driving environment such as better lighting helps the driver of the following vehicle notice objects in front more clearly, while more consistent 
geometry can improve speed coordination in road sections with different road features. Besides, it is also recommended to make changes in design standard for shoulder width in underground expressways to spare $0.5 \mathrm{~m}$ on right shoulder and install eye-catching reference on right roadside.

\section{References}

[1] The Official Website of The Massachusetts Department of Transportation: http://www.massdot.state.ma.us/highway/TheBigDig.aspx

[2] Singapore Land Transport Authority: http:/www.lta.gov.sg/content/ltaweb/ en/roads-and-motoring/projects /kallang-paya-lebar-expressway-kpe.html

[3] Guojun, H. Research on Scope and Layout of Expressways in China. Southeast University, Nanjing, China, 2005.

[4] Wanlian, L., Lianbo Z. \& Chunmei, Q., Sustainable development of urban transport and underground expressway. Journal of Changsha Transport College, 22(3), pp. 44-48, 2006.

[5] Finn, H. A. \& Guro, R. Studies on traffic accidents in Norwegian road tunnels. Tunnel Safety, 15(1), pp. 3-11, 2000.

[6] Yue, W., Junhua, W., Shouen, F. \& Can, C. Research on Traffic Sign Schemes for Underground Expressway. Proc. of 11th Int. Conf. of Chinese Transportation Professionals (ICCTP), eds. Y. Yin, Y. Wang, J. Lu \& W. Wang, American Society of Civil Engineers, 2011, pp. 3144-3153.

[7] Tiejun, Z. Zhengzheng, T. \& Nan, S. Impact of road safety project on operating characteristics of vehicles in curved downhill sections. Journal of Highway Transportation Technology, 24(1), pp. 130-133, 2007.

[8] Min, H. Study of Car-following Theories. Beijing University of Technology, Beijing, China, 2000.

[9] Terumitsu, H. \& Tetsuo Y. Traffic Safety Analysis in underground urban expressway using a driving simulator. Journal of the Eastern Asia Society for Transportation Studies, 2003(5), pp. 2592-2606. 